A N N A L E S Annales de Bretagne et des Pays de l'Ouest

\title{
FICHOU, Jean-Christophe, Les pêcheurs bretons durant la Seconde Guerre mondiale (1939-1945)
}

\section{Christian Bougeard}

\section{OpenEdition}

\section{Journals}

Édition électronique

URL : https://journals.openedition.org/abpo/1973

DOI : $10.4000 / a b p o .1973$

ISSN : 2108-6443

\section{Éditeur}

Presses universitaires de Rennes

\section{Édition imprimée}

Date de publication : 10 avril 2011

Pagination : 210-214

ISBN : 978-2-75351410-2

ISSN : 0399-0826

\section{Référence électronique}

Christian Bougeard, «FICHоU, Jean-Christophe, Les pêcheurs bretons durant la Seconde Guerre mondiale (1939-1945) ", Annales de Bretagne et des Pays de l'Ouest [En ligne], 118-1 | 2011, mis en ligne le 04 mai 2011, consulté le 08 décembre 2022. URL : http://journals.openedition.org/abpo/1973 ; DOI : https:// doi.org/10.4000/abpo.1973 
du 25 juin 1915, il note ces quelques mots : « journée calme et tranquille; on ne se croirait vraiment pas à la guerre » (p. 53).

Mobilisé en tant que sous-officier, Paul Cocho décroche ses galons d'officier au feu, signe d'une acquisition "sur le tas » de cette "culture professionnelle » des combattants qui, indubitablement, distingue ceux qui connaissent la réalité du champ de bataille des autres ${ }^{4}$. Ainsi note-t-il en janvier 1916, à propos d'un commandant nouvellement arrivé dans son unité, qu'il a " encore tous les enthousiasmes et toutes les naïvetés de ceux qui n'ont pas vu vraiment le feu » (p. 89), propos qui, évidemment, peuvent se rapporter à sa propre acculturation à la guerre moderne : nombreux sont les passages de ses carnets qui en témoignent.

Capturé en mai 1918, Cocho n'en poursuit pas moins leur rédaction, révélant ainsi l'importance de l'écrit pour lui. Dans ses deux derniers carnets, il décrit tout d'abord les conditions de vie d'un officier captif des lazarets allemands - en raison d'une blessure par éclat d'obus au bras gauche reçue lors de la vaste offensive allemande sur le Chemin des Dames - avant de s'intéresser à la situation de l'Allemagne, en pleine révolution à la fin de l'année 1918. On pourra certes objecter que ce témoignage, émanant d'un officier, n'est sans doute pas le plus représentatif de la condition des prisonniers de guerre bretons pendant le Premier Conflit mondial. II n'en demeure pas moins extrêmement riche, tout particulièrement en ce qui concerne le périple du retour au pays, une fois la guerre terminée, et devrait satisfaire le lecteur avide de matériaux pour une histoire renouvelée des Bretons dans la Grande Guerre.

On saura donc particulièrement gré à Françoise Gatel et Michel Doumenc d'avoir transcrit ces "carnets de guerre et de prisonnier », conférant par la même occasion à Paul Cocho une "gloire littéraire » posthume que lui-même n'aurait sans doute jamais soupçonnée.

Erwan LE GALL

FICHOU, Jean-Christophe, Les pêcheurs bretons durant la Seconde Guerre mondiale (1939-1945), Rennes, PUR, coll. « Histoire», 2009, 343 p.

Spécialiste reconnu d'histoire maritime, travaillant sur le temps long (18501980), I'histoire des techniques et l'histoire sociale (les phares et balises, les gardiens de phares, les conserveurs et entreprises de conserveries de sardines), Jean-Christophe Fichou est un inlassable découvreur d'archives. Dans cet ouvrage, il s'est attaqué à la situation des " pêcheurs bretons durant la Seconde Guerre mondiale ». Insistant en introduction sur l'absence d'études historiques et la méconnaissance du monde de la pêche sous l'Occupation et s'appuyant sur les fonds des archives inédites des administrateurs de I'Inscription maritime et beaucoup d'autres sources, il pourfend quelques idées reçues sur les limitations (la limite des trois milles), les nombreuses interdictions de sortie en mer imposées par l'occupant et le déclin de la pêche qui aurait été sinistrée pendant la période. Sur le fonctionnement de ce secteur d'activité économique et la vie quotidienne des pêcheurs, l'ouvrage de Jean-Christophe Fichou apporte beaucoup à notre connaissance " des années noires » car même si elle est centrée sur la Bretagne son analyse dépasse largement les limites régionales. II précise le rôle et les politiques de Vichy ainsi que la réalité du contrôle des diverses autorités allemandes

4. L'expression est de Prost, Antoine et Winter, Jay, Penser la Grande Guerre. Un essai d'historiographie, Paris, Seuil, 2004. p. 127. 
moins rigoureux qu'on ne l'a dit parfois car l'occupant a besoin de poisson pour nourrir ses troupes.

Construit en sept chapitres, l'ouvrage s'ouvre par un tableau de la situation en 1939-1940 lors de la déclaration de guerre en rappelant l'organisation de l'administration des pêches maritimes relevant du ministère de la Marine marchande d'ailleurs placé sous l'autorité de ministres bretons (Louis de Chappedelaine, député de Dinan 2, puis Alphonse Rio, élu du Morbihan). En 1939 la Bretagne compte 34000 (57\%) des 60000 inscrits maritimes pêcheurs français exerçant leur activité dans une centaine de ports, répartis en 18 quartiers qui abritent plus de 8500 bateaux dont encore $53,7 \%$ à voile. Cette région représente $40 \%$ (en tonnage et en valeur) des prises françaises faisant vivre 150000 personnes dans l'industrie des pêches. En dépit de la mobilisation et de la réquisition de navires, la pêche reprend rapidement pendant la drôle de guerre et, alors que le rationnement s'élabore sans vraiment être appliqué, elle devient une activité lucrative surtout pour les crustacés dont le prix de vente double ou triple avant même l'occupation allemande. Déjà, les produits de la mer prennent le relais de la viande sur certaines tables les jours de fermeture des boucheries.

Dans le cadre de la Révolution nationale, dès l'été 1940 et surtout au temps de l'amiral Darlan, vice-président du Conseil de février 1941 à avril 1942 (retour de Laval), les officiers de la Marine nationale occupent de fortes positions de pouvoir dans les administrations de l'Etat français. Le secrétariat d'Etat à la Marine est une pièce maîtresse d'un régime, dominé par des militaires, qui veut s'appuyer sur le monde de la mer comme il veut le faire avec la paysannerie pour réorganiser la société française à la faveur de la défaite. Mais le nouveau régime doit gagner la confiance de marins pêcheurs souvent méfiants à l'égard de la Royale. Or, ce milieu de tradition républicaine votait très à gauche, notamment pour le PCF dans les années 1930 dans les ports du Finistère sud. Cet élément de la culture politique est insuffisamment souligné pour comprendre l'échec des politiques vichystes. II n'en reste pas moins que Vichy va choyer cette population tout en essayant d'encadrer les jeunes inscrits dans des Chantiers de jeunesse de la Marine échappant au contrôle du secrétariat d'Etat à la jeunesse et dans 14 écoles d'apprentissage maritime (EAM) ouvertes sur les côtes de France en octobre 1941, poursuivant ainsi la politique de la Troisième République. Pour réussir cette expérience et dispenser une formation conforme à l'idéologie de la Révolution nationale, la Marine s'appuie sur la Jeunesse maritime chrétienne (JMC), un mouvement d'action catholique fondé en 1930. Mais comme dans beaucoup d'autres projets de Vichy, les résultats sont limités (de 400 à 700 jeunes par an) et les résistances du milieu fortes.

En revanche, les pêcheurs bénéficient de nombreux avantages matériels dont la part réservataire qui leur permet de vendre directement, et à bon prix, une partie importante de leurs prises, ce qui alimente les nombreux marchés parallèles (gris et noir) que les autorités cherchent à combattre. Souvent, ce sont les Allemands euxmêmes qui achètent court-circuitant le marché officiel à prix taxés. En ces temps de pénuries et de paupérisation croissantes des salariés, ces «privilèges » des pêcheurs sont de plus en plus mal perçus, notamment par des fonctionnaires contraints de se serrer la ceinture. Avec une forte dissimulation des prises et des statistiques truquées, l'attitude des pêcheurs n'est guère différente de celle de nombreux agriculteurs. À cet égard, dans les zones littorales il aurait été intéressant de regarder s'il n'y avait pas de circuits d'échanges, voire de troc entre les uns et les autres.

Le chapitre III analyse la politique des pêches maritimes en confirmant pour ce secteur ce que l'on sait déjà de l'expansion bureaucratique (multiplication des organismes administratifs et économiques) et réglementaire sous Vichy. L'accumulation de textes et de circulaires ne sert qu'à masquer l'impuissance croissante d'un Etat 
central qui ne contrôle pas la situation en zone occupée où l'armée allemande impose son ordre en fonction de ses besoins insatiables en denrées alimentaires et en hommes. Vichy veut centraliser les organismes professionnels en les intégrant comme pour les autres branches dans des comités d'organisation (CO). Aspect méconnu, Jean-Christophe Fichou met à jour la création le 13 mars 1941 de la Corporation nationale des pêches maritimes, sous Darlan, après celle le 2 décembre 1940 de la Corporation paysanne dirigée par le grand agrarien et responsable de l'Office central de Landerneau, Hervé Budes de Guébriant. Cet organisme de 36 membres nommés par le régime, présidé par le vice-amiral d'escadre Le Bigot " un marin et un chef », tient sa séance inaugurale le 27 août 1941 en présence de l'amiral Auphan. Cette Corporation s'appuie sur des responsables professionnels et syndicaux catholiques d'avant-guerre dont quelques uns basculeront dans la résistance en 1943 ou 1944 (l'ex-député du Morbihan Firmin Tristan). Le milieu patronal des conserveurs, en quête de revanche sociale sur 1936, se rallie d'emblée à la Révolution nationale (chapitre $\mathrm{VI}$ ), mais il doit rapidement constater que les organismes vichystes servent surtout à répartir les pénuries. Les plans de modernisation ou d'adaptation (propulsion aux gazogènes) de la flottille et des ports ne peuvent être menés à bien, surtout lorsqu'à partir de 1942 l'organisation Todt réquisitionne tous les moyens disponibles. Dans ce domaine comme dans d'autres, Vichy échoue à diriger ce secteur économique car l'inflation et les marchés parallèles font peu de cas de la fixation des prix et de la répression des fraudes. Et l'administration locale répond de moins en moins aux ordres.

De toute façon, les pêcheurs n'en font souvent qu'à leur tête, aussi bien dans le respect des zones de pêche délimitées par l'occupant, d'où des incidents et parfois des morts quand les Allemands tirent sur les bateaux (à partir de 1942, chapitre VII), que dans la commercialisation directe de la part réservataire plus lucrative. Alors que la pêche reprend dès l'été 1940 - l'année 1940 aurait été la meilleure de la décennie en terme de prises - elle se poursuit jusqu'à la libération, y compris pendant l'été 1944, avec la bénédiction des Allemands. Tous les chiffres fournis sont faux: en mai 1942, les agents du Contrôle économique de Vichy estiment que dans certains ports la moitié des prises échappe au circuit officiel, celui du Ravitaillement général à prix taxés. Pour les consommateurs, les produits de la mer disparaissent des étals, y compris dans les villes de Bretagne, sans parler des campagnes, alors que les restaurants parisiens et autres servent leurs clients allemands et les privilégiés français en homards, huîtres et poissons nobles. La situation alimentaire ne cesse de se dégrader longtemps après la Libération. Jean-Christophe Fichou n'insiste sans doute pas assez sur les perceptions de cette situation vécue de pénuries, notamment par les salariés et les citadins, ce qui permettrait de mieux comprendre les représentations partiales ou incomplètes de ce secteur d'activité économique. On n'a en effet retenu que les interdictions et les limitations de sortie en mer sous l'Occupation alors que la situation est autre. Et c'est tout le mérite de ce livre de montrer l'ensemble des aspects d'une société maritime qui en fait s'adapte plutôt bien aux contraintes du temps.

Les chapitres IV et V précisent cette réalité. De 1940 à 1942, la pêche est d'autant plus soutenue que les Allemands y trouvent un grand intérêt pour nourrir leur population (conserves de poissons) et leurs troupes en garnison. Parallèlement, Vichy a aussi intérêt à l'essor de la pêche pour répondre aux carences alimentaires de la population en dépit des risques encourus par les pêcheurs du fait des attaques britanniques en mer. En outre, les côtes bretonnes donnent lieu à des évasions par mer, à la mise en place de réseaux d'évasion vers l'Angleterre provoquant des mesures de rétorsion des Allemands, à des comportements de désobéissance et de rejet marqués d'autant plus que la dissidence gaulliste (Français libres) concerne 
de nombreuses familles de marins bretons. Jean-Christophe Fichou souligne que les pêcheurs résistants sont une poignée par rapport à l'ensemble du milieu et que beaucoup préfèrent continuer leur métier. Certes. Mais, à notre sens ce n'est pas seulement une question de nombre : l'auteur ne prend pas suffisamment en compte les travaux récents qu'il cite portant sur l'ambivalence des comportements (Pierre Laborie) ou les rapports des résistants à la société (voir les réflexions de François Marcot sur le couple fonctionnalité/intentionnalité) même s'il rappelle la participation des milieux maritimes aux combats de la libération. C'est une question d'échelles et de focale.

Le chapitre V consacré à la " production et [à la] consommation » étudie précisément les formes et les difficultés de la pêche montrant que l'année 1942 est la meilleure pour la pêche à la sardine (en tonnage), que les pêches à pied et la conchyliculture ne cessent de se développer pour pallier les carences alimentaires, si bien qu'on ne respecte plus aucune règlementation au risque de détruire la ressource. Face aux pénuries croissantes, les autorités françaises en sont réduites à fermer les yeux (taille, qualité), les mareyeurs ne sont guère regardants et les acheteurs n'ont pas le choix. Comme l'écrit Jean-Christophe Fichou : "On mange tout, on pêche tout $»$, y compris l'immangeable.

Spécialiste de I'histoire des conserveurs (voir son HDR soutenue à I'UBO), I'auteur dresse dans le chapitre VI un tableau précis de la conserverie de poisson, secteur florissant et fort rémunérateur pour les usiniers dont le nombre d'usines sur la côte atlantique passe de 169 en 1939 à 204 en 1942 et 150 en 1944. Dès 1939, dans le cadre de la mobilisation, l'Etat achète sans discuter la production de conserves pour l'armée à des prix intéressants tout en réprimant les rares grèves des ouvrières protestant contre le blocage de leurs bas salaires pendant le drôle de guerre, pour le plus grand profit d'un patronat très dur. A Hennebont, en novembre 1939, des " meneuses » sont même condamnées à de la prison ferme. Dès l'été 1940, les Allemands prennent le relais en achetant à bon prix, grâce à l'indemnité journalière de guerre versée par la France, la plus grande partie des stocks de conserves (sardines, thons, légumes) et de la production. Par le système de contrôle de Vichy (CO) et un organisme d'achat qu'ils contrôlent, les conserveurs se réservent de 75 à $90 \%$ des prises qu'ils vendent ensuite transformées aux Allemands alors que la conserve est un produit de luxe. Appuyant pourtant le régime de Vichy, les patrons conserveurs refusent de répondre aux demandes d'enquêtes de l'administration française sur leurs stocks afin d'avoir les mains libres et échapper à d'éventuelles réquisitions pour nourrir les Français. En revanche, leur demande volontaire de classement en usines $V$ Betriebe puis $S$ Bebriebe leur permet de jouir de la protection de l'occupant et d'être prioritaires en matières premières (boites de conserves) et en énergie. La collaboration technologique des firme allemandes et françaises est engagée notamment sur les questions de congélation (accords de mars 1941 signés par une douzaine de firmes françaises dont les plus importantes : les sociétés Amieux, Saupiquet, Cassegrain...). C'est donc un commerce libre, payé rubis sur l'ongle par l'occupant, car ces conserveries n'ont jamais été réquisitionnées; un seul industriel, Noël Larzul, a choisi en 1940 de fermer ses portes pour ne pas travailler pour l'occupant. Aux produits de luxe (champagne, vins notamment du bordelais) achetés massivement par les Allemands pillant méthodiquement l'économie française, l'auteur ajoute les conserves de poisson devenues introuvables ou inabordables pour les Français ordinaires.

Même si dans la solide bibliographie, les études sur l'épuration figurent en bonne place, on ne peut que regretter l'absence d'une analyse de l'épuration économique des conserveurs et des conserveries. Les sources n'étaient sans doute pas disponibles et il aurait été utile de l'indiquer. On peut regretter aussi quelques 
coquilles et erreurs, tirées parfois de sources non rectifiées : aéroport de RennesSaint-Jean pour Saint-Jacques (p. 83), « départ de Pierre Laval en octobre 1940 » alors qu'il est renvoyé par Pétain le 13 décembre (p. 154), affaire de l'évasion par du Vicking (sic), (p. 148), René Meyer pour René Mayer, commissaire du CFLN et du GPRF (p. 306).

Au total, ce nouveau livre de Jean-Christophe Fichou comble un vide historiographique sur la France des Années noires. Sa connaissance des archives, du milieu maritime, des techniques apporte de multiples éclairages qui dépassent largement le cadre breton.

Christian BOUgeARD

FISHMAN, Sarah, La bataille de l'enfance. Délinquance juvénile et justice des mineurs en France pendant la Seconde Guerre mondiale, Rennes, PUR, 2008, 323 p., $20 €$.

Il faut se féliciter de la publication par les Presses Universitaires de Rennes de la traduction française du livre de Sarah Fishman, The Battle for Children, paru en 2002 à Harvard University Press ${ }^{5}$. En effet, cet ouvrage marque un jalon en matière d'historiographie de la jeunesse de la Seconde Guerre mondiale, ou plus précisément $d$ '« une » jeunesse dans la Seconde Guerre mondiale : la jeunesse délinquante. La réflexion brillamment menée par Sarah Fishman dans ce livre part d'un constat : perceptible dans la totalité des pays européens lancés dans le conflit, l'augmentation vertigineuse des chiffres de la délinquance juvénile est particulièrement spectaculaire en France, où la guerre créa une situation sans précédent en matière de criminalité. Professeure associée en histoire à l'Université de Houston, l'auteure poursuit dans La bataille de l'enfance sa quête de la famille durant le second conflit mondial entreprise avec une thèse remarquée sur les femmes de prisonniers de guerre $^{6}$. Cette nouvelle publication est d'autant plus en lien avec la précédente que, pour bon nombre des observateurs de l'époque, l'absence prolongée des pères prisonniers (près de 600000 soldats français capturés en juin 1940 et envoyés dans les camps de prisonniers en Allemagne étaient des pères de famille) aurait été la cause de l'augmentation de la délinquance juvénile.

Cette étude entend opérer la jonction entre d'un côté une histoire politique (au cœur des années de guerre) et, de l'autre, une histoire sociale qui interroge une catégorie assez silencieuse de I'histoire (à fortiori dans un contexte guerrier), celle des enfants et particulièrement ici, ceux qui ont eu à faire à un moment donné à la justice. Mais surtout, toute la teneur de cet ouvrage est sous-tendue par un objectif, mettre au jour un nouvel aspect méconnu du gouvernement de Vichy, dans ce qu'il a de réformateur quant au système judiciaire des mineurs. En effet, Sarah Fishman s'attache à confirmer l'idée que c'est, paradoxalement, le régime de Vichy, gouvernement profondément autoritaire, anti-démocratique, paternaliste et corporatiste, qui, prenant la mesure de la nécessité d'agir en ce domaine, entreprend de réformer la justice des mineurs, afin de réduire la nature répressive de ce système.

5. Sarah Fishman, The Battle for Children. World War II, Youth Crime and Juvenile Justice in Twentieth-Century France, Cambridge, Mass., Harvard University Press, 2002 - traduit de l'anglais (USA) par Johann Maunet et révisé par Jacqueline Sainclivier.

6. Sarah FishmAn, Femmes de prisonniers de guerre 1940-1945, Paris, L'Harmattan, 1996, traduction de We will wait : Wives of French prisoners of war, 1940-1945, Yale University Press, New Haven and London, 1991. 